DOI: $10.46523 /$ jarssc. 05.01 .09

Multidisciplinary, Open Access

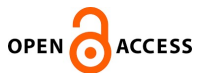

\title{
Understanding the trends in Health Communication: Mobile Health Applications and Accredited Social Health Activists (ASHA)
}

\author{
Kamna Kandpal \\ Research Scholar, Amity School of Communication, Amity University, Noida \\ E-mail: kamna.kandpal@s.amity.edu \\ Dr. Piyashi Dutta \\ Assistant Professor, Amity School of Communication, Amity University, Noida \\ E-mail:pdutta@amity.edu \\ Dr. P. Sasikala \\ Dean \& Professor, Department of New Media Technology, \\ Makhanlal Chaturvedi University, Bhopal \\ E-mail: sasikala@mcu.ac.in
}

\begin{abstract}
The spread of mobile technology in the health care domain has created a new field of e-health. eHealth has customized and tailored services for less privileged people. This paper discusses mHealth applications that use mobile technology to disseminate health information to patients. The government of India launched mHealth Applications that run in certain states under various health programs. The paper analyzes rapid advancements in technology that have made it possible to quickly monitor health through the use of mHealth applications. Accredited Social Health Activists (ASHA) use mHealth applications to counsel women in context to maternal Health.The study examines the Health Applications launched to aid ASHA's especially for counseling, point-of-care services, health information provision, and data monitoring. The paper presents the trends in context to health communication and widespread use of mHealth applications to cater women's health issues The trends in health communication involve patient health monitoring through increased patient and healthcare provider interaction, and $24 \mathrm{X} 7$ healthcare support. The analysis shows Healthcare through mobile Applications has improved communication, transparency, and healthcare services dissemination across boundaries. Digital Empowerment to health workers has brought a sea change in primary and tertiary care. It is found that Real-time data sharing has digitally empowered ASHA workers and reduced their burden of data-related work.
\end{abstract}

Keywords: Health Communication, mHealth, Accredited Social Health Activists, Reproductive Health, Digital Healthcare 
Journal of Advance Research in Science and Social Science (JARSSC)

Official Publication of Indian Mental Health \& Research Centre

DOI: $10.46523 /$ jarssc. 05.01 .09

Multidisciplinary, Open Access
ISSN: 2582-2004

Volume 05, Issue 01

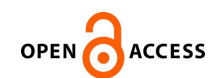

Crossref

\section{Introduction}

The National Rural Health Mission (NHRM) was established in 2005 to communicate the health demands of rural communities. The aim of the mission is that there have to be ASHA workers in each village. ASHA workers are regarded as the health communicators of the region they serve. The Ministry of Health and Family welfare defined ASHA as health activists who create awareness on health and mobilizes the community towards health planning as well as accountability towards health services. ASHA workers are the initial point of contact between the health officials and the community women. The training imparted to them decides the course of action in the field. The main tasks performed by community health workers include 1) Motivating women to give birth in the hospitals, 2) encouraging family planning through surgical sterilization, 3) treating basic illness, 4) keeping demographic records, 5) Improving Village Sanitation.

Srivastava et al., (2016) mentioned the role of healthcare workers as effective in building health capacity in context to Health communication. ASHA workers are strengthened through the training imparted to strengthen communication skills to get desired results.

They are health communicators and perform the tasks of communicating health communication to community women. However, Health Communication delivery needs to be strengthened and effective measures need to be adopted. Therefore, Health Communication can be defined as the multifaceted and multidimensional approach that involves health-related information to influence, support, and engage individuals, policy-makers, health professionals, and the public to adopt, sustain or introduce a behavior or practice that improves health outcomes.

Health Communication is defined as the niche that influences the decisions of the people and allows professionals to use communication strategies. It is the practice and study of communicating promotional health information. The main purpose is to influence health choices through health literacy by disseminating health information. Schiavo, (2007) defined Health Communication as "1) Exchange of information between two individuals, 2) Message - Use of message and written form, 3) Act of Communicating - the actions involved in imparting the message, 4) Rapport- sympathy and a sense of mutual understanding,5) Accesschannel for communicating" (p.4). It is essential, firstly it enhances the knowledge of the audience and awareness on health issue, secondly, it influences attitudes and behavior towards 
Journal of Advance Research in Science and Social Science (JARSSC)

Official Publication of Indian Mental Health \& Research Centre

DOI: $10.46523 /$ jarssc. 05.01 .09

Multidisciplinary, Open Access
ISSN: $2582-2004$

Volume 05, Issue 01

the health issue, Third, it increases demand for health services, and lastly, it demonstrates behavior changes to public health outcomes.

Kohli et al. (2015) mentioned the success of the National Rural Health Mission depends on the training and performance of the health workers. ASHA workers have been regarded as grass root level workers. They are often over-burdened with work It addresses the issues that ASHA's face while working in the fields to assess the modifications and actions required. The research question arises as to How can health care workers be empowered so that they deliver their services effectively? Digital Empowerment to ASHA's can transform grassroot healthcare and hence are regarded as Digital disruptors. Training and connecting with them can bring a sea change in primary and tertiary care. Although, ASHA's are digitally empowered to some extent. The spread of mobile technology has created a new field called "mHealth" that provides customized and individually-tailored service to less privileged people.

Interactive Health Communication has been defined as an individual as a caregiver or professional through the communication technology via an electronic device to transmit health information on health-issue (Gustafson et al.,1998, p.1264). This argues that Telemedicine has been viewed as the solution to bridge the rural-urban digital divide and the supply of services. It solved the three main issues in healthcare that included awareness, access, and affordability. India has approximately 9,00,000 ASHA workers and performs several duties in context to maternal and Child Health. The National Digital Health Mission (NHDM) launched on $15^{\text {th }}$ August 2020 enables bridging the stakeholders of the healthcare ecosystem to bridge the existing gaps. The improvement in the healthcare delivery system enhances motivation and gains the community's trust.

India experienced huge growth in the telecommunication industry, with mobile phones reaching rural areas. The availability and use of mobile phones by the majority of health workers provide an opportunity if technology helped in reducing the burden of Female Health workers' (FHW's) productivity and improve the quality of services. Hence, Technological adoption has bridged the digital divide. The use of smartphones has enabled them to act as Digital Health Agents to deliver solutions in context to healthcare to the target audience.

The digital revolution brought changes in the healthcare sector with technology working in every process. The concept of eHealth through the use of ICT ensured management and 
Journal of Advance Research in Science and Social Science (JARSSC)

Official Publication of Indian Mental Health \& Research Centre

DOI: $10.46523 /$ jarssc. 05.01 .09

Multidisciplinary, Open Access
ISSN: 2582-2004

Volume 05, Issue 01

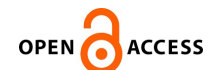

Crossref

optimization in context to diagnosis for efficient patient care. According to statistical data Portal, the eHealth Industry showed a rise with \$14.6billion investments globally.

The Report on WHO defined eHealth as safe and cost-effective use of ICT in context to health and health-related fields. eHealth encloses multiple interventions that include telehealth, telemedicine, mobile health (mHealth), electronic records, and artificial intelligence. The eHealth role has been recognized as important in achieving health such as SDG (Sustainable Development Goals) and Universal Health Coverage (UHC).

Rudolph et al., (2008) in the study E-Health and its impact on Medical Practice mentioned the goals of e-Health activities includes 1) Increase in access to health care, 2) Clinicians expanded capacity, 3) Patients better outcomes, 4) Cost-care Reduction, 5) Heath care Personalized, 6) Increase in Participation of Patient and self-management of Health, 7) Development of virtual practices, 8) Increased work productivity in context to reduced work time. e-Health Potential benefits in context to patient \& physician that involves firstly, Increasing access of the patient to high-quality health care through established relationships that makes health care guidance available with no face to face visit, Secondly, Improvement in patient-physician communication by using the technological strategy, Thirdly Enhancing Patient Satisfaction by improving access to quality healthcare from health care team and Physicians, Lastly Enable virtual teams contribution towards enhanced patient processes.

In this context, this paper aims at understanding the applications launched to help the ASHA workers and the emerging trends in Health Communication. It discusses the mHealth apps that improve efficiency in the workload.

\section{METHOD}

A systematic review was conducted utilizing a literature search in digital health databases and other information sources in context to Mobile health applications. The apps were classified based on trends in context to health communication and digital empowerment to Accredited Social Health Activists (ASHA).

\section{Overview on Digital Health and Healthcare}

Digital India initiated various e-Gov initiatives named as eHealth division by the Ministry of Health and Family Welfare (MoHFW) of Health sectors in India. e-Health is defined as the use of ICT (Information and Communication Technology) in India. Integrated Health serves the 
Journal of Advance Research in Science and Social Science (JARSSC)

Official Publication of Indian Mental Health \& Research Centre

DOI: $10.46523 /$ jarssc. 05.01 .09

Multidisciplinary, Open Access
ISSN: 2582-2004

Volume 05, Issue 01

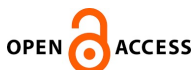

Crossref

needs of the stakeholders by 8 percent of the GDP. The e-Health initiatives had the vision to deliver better health outcomes in terms of, 1) access 2) Quality 3) Affordability 4) Efficient monitoring of health entitlements to its citizens.

E-health is defined as facilitating, promoting, and empowering families, individuals, and communities through the use of information and communication technology (ICT). E-health includes following, firstly Patient records in electronic forms (include assessment and care planning, patient schedules, clinical communications), secondly electronic communication with professionals and patients (telephonic support, email, SMS texts), thirdly telehealth (remote monitoring and video consultations), fourth information Management includes reusing data to improve care, health care, and patient information), Fifth Information governance includes covering confidentiality, data protection, and system security and Lastly, Personal Data records includes a repository of information considered relevant to the health, development, and welfare of an individual.

The study 'Role of Mobile Phone in Female health work routine conducted as the field study in Mysore, Karnataka' stated that Project Drishti was initiated in Karnataka involved developing android based mHealth applications for FHW and evaluating the impact on maternal and Child Health indicators. It discusses various aspects firstly Use of Mobile Phones in FHW's work, secondly Access to Mobile Phones, Thirdly Knowledge and ability to handle mobile, and lastly Mobile usage among mothers and their mode of communication (Murthy \& Vijayaraman,2012).

Desai (2017) in the study 'e-Health in India' stated services and tools using ICT that can improve prevention, diagnosis, treatment, management for purposes in context to health. This emphasizes ICT as an important tool in the effective management of health issues.

Zaman (2017) in the study 'contexts and opportunities of e-Health Technology in Medical care' stated in context to health care provision e-Health is considered as a promising vehicle. For the implementation of m-Health approaches improvement in context to e-Health, technology would be beneficial for patients. mHealth is referred to as a subset of e-Health. m-Health Technologies can bridge the gaps in context to patient and healthcare support. It also argues that a patient-centered health care system involves incorporating technology in context to medical care. 
Journal of Advance Research in Science and Social Science (JARSSC)

Official Publication of Indian Mental Health \& Research Centre

DOI: $10.46523 /$ jarssc. 05.01 .09

Multidisciplinary, Open Access
ISSN: $2582-2004$

Volume 05, Issue 01

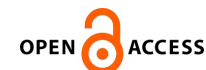

Crossref

e-Health benefits can be defined in the form of 10e: 1) Efficiency-e-health increases efficiency in healthcare. It leads to enhanced communication between healthcare providers and patients. 2) Enhancing Quality- Increasing efficiency leads to increased quality but also reduces costs. E-Health enhances the quality of healthcare by allowing comparisons, involving consumers for quality assurance. 3) Evidence-based - E-health interventions are based on evidence as their efficiency is calculated by rigorous evaluation. 4) Ethics- e-Health involves new challenges to ethical issues such as informed consent, Privacy, and Equity issues, and patient-physician interaction 5) Extending- The scope of healthcare extends to boundaries both in a conceptual and global sense.

The initiatives are available to medical facilities from any part of the world all-time through web services, SMS, and mobile services. Patient information exchange can be made through Online medical consultations, online medical records, online medical supply, and Pan-India. The related technologies and the internet have significantly extended healthcare scope beyond its traditional boundaries (Esyenbach, 2001,pg. 20). This argues that Telemedicine, artificial intelligence, and AI-enabled medical devices transform medical healthcare and reshape our interaction with health professionals. e-Health emerged as a field of the intersection of public health, medical informatics, health services referrals, and information delivered through related technologies and the Internet. The new concept of information and communication technology provides digital health interventions to improve the quality of life. Global concerns ranging from maternal and child mortality, health systems, and related technology continue to grow and evolve. Health care informatics is defined as employing technology and data to improve patient health as well as the quality of care. Healthcare professionals integrate new programs to look for areas of improvement and integrate new technology in the health sector.

(Karahainko,2020) in the study 'The innovative future of Healthcare with the mHealth apps 'mHealth Technologies have a lot of core benefits firstly convenience: Mobile Health Technology especially to the elder population and children provide comfort to the patients. Direct access to health care professionals and health care facilities is available with just one tap on their mobile devices. Secondly, High Efficiency - mHealth Applications increases the efficiency of work and play a positive role in context to accurate information, and reduce the burden of manual documentation., Thirdly, Convenient Documentation- Health Professionals spend a lot of their time on monotonous paperwork, and online medical records can be accessed 
Journal of Advance Research in Science and Social Science (JARSSC)

Official Publication of Indian Mental Health \& Research Centre

DOI: $10.46523 /$ jarssc. 05.01 .09

Multidisciplinary, Open Access
ISSN: 2582-2004

Volume 05, Issue 01

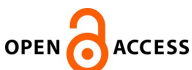

Crossref

in the future. Fourthly, Improved ROI: mHealth Applications have built a reliable name for different applications in different parts of the country for patients' feasibility and improvement in quality care.

Arya (2019) conducted the study in Uttarakhand to test the rural health service delivery efficiency and its impact on rural health services in context to quality. It also explores how ICT could be used as a tool to facilitate networking with rural healthcare workers. The findings stated use of Information and Communication technology has the potential to improve public healthcare system performance. It could transform the quality and access of health services. The research question arises as to How it would transform the system? The argument that transformation of the traditional approach to Internet-based medical health (mHealth) has brought a transition in healthcare awareness and empowered Health workers. The Internet of Medical things (IoT) refers to applications connecting to IT systems and a combination of medical devices that uses networking technologies. IoT uses telemedicine technology to improve communication between caregivers and patients.

The research question arises How do digital Healthcare shape the future of Health? The impact created through digital healthcare improves the diagnosis, 2) It facilitates quick delivery of medical healthcare,3) It advances personalized care. 4) It increases financial outcomes, 5) It empowers healthcare workers and health professionals, 6) It improves drug discovery, and 7) It accelerates clinical development.

Chib et al., (2012) in the study 'ASHA: The hope of information communication technologies in rural Healthcare' stated that ICT as a healthcare tool has four key benefits that bring an increase in knowledge leading to healthcare outcomes. Firstly, opportunity Producer plays an important role in health service delivery as a prevention tool in earlier diagnosis and intervention (ITU, 2010, Olla \& Tan, 2008), secondly, Capability Enhancer-Mobile Phones increase task handling efficiencies and make work more organized, convenient, and completed. Community Health workers are equipped with providing data, monitoring trends, and leading to improved program performance (Chib, 2010). This emphasizes the Mobile Health applications devised to aid the community health workers through digital empowerment and argues on the traditional concept of healthcare services. 
Journal of Advance Research in Science and Social Science (JARSSC)

Official Publication of Indian Mental Health \& Research Centre

DOI: $10.46523 /$ jarssc. 05.01 .09

Multidisciplinary, Open Access
ISSN: $2582-2004$

Volume 05, Issue 01

\section{Overview on Mobile Health applications: Usability and Applicability}

Digital Health or digital healthcare is multidisciplinary in its aspects from the intersection between healthcare and technology. Digital health application involves a transformation by incorporating software and hardware services in the healthcare field. Digital health includes mobile health (health) apps, wearable devices, telemedicine, telehealth, electronic medical records (EMR's), and personalized medicine. Digital healthcare plays a very essential role in the healthcare domain.

The digital mobile application is regarded as an easy-to-use interactive guidance system and full-fledged that enables community health workers to assist the community women. Mobile phones use open-source software that is equipped with multimedia aids to support patient assessment, counseling, early identification, treatment, and rapid referral of pregnancy, postpartum and newborn complications. The ASHA application registers each new mother, enters patient data into the phone, and walks through checklist, questions, and educational prompts to identify current issues. It also determines whether prior treatment was followed and share new counseling tips to pregnant women based on their stage of pregnancy.

2.1) e-ASHA - The United Nations Children's Fund with the assistance of IIT, Jodhpur designed an innovative approach to provide rural health workers with the technology that made the communication effective. The innovative approach is called the e-ASHA. It consists of a tailor-made application that is an efficient way of monitoring, identifying, and tracking mother and Child health. This concept was field-tested and approached in the difficult-to-reach cluster of remote villages. The various health applications that have been launched to aid the ASHA workers.

2.2) mSakhi application- The mobile application for frontline health workers has received recognition in the context of healthcare. mSakhi is an online android application developed for frontline health workers. It has an electronic-enabled record system that helps the health workers up-to-date training and gain access that is easier to understand. The uses of the mSakhi are as follows firstly, it allows the ASHA workers to stay in contact with supervisors secondly, smartphones to update their skills, third, it reports in communities' important data related to health issues. Reach and access of the mSakhi application - The initiative is reaching 12,000 frontline workers in five districts led by the government of Uttar Pradesh. This app will soon be available in Uttarakhand and Jharkhand. mSakhi is based on the National Rural Health 
DOI: $10.46523 /$ jarssc. 05.01 .09

Multidisciplinary, Open Access

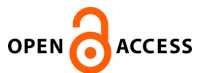

Mission curriculum for ASHA and includes messages, audio recordings, and illustrations on prenatal care, immunization, and nutrition are in the local language.

2.3) Pragati- Peer learning application - It is a peer learning mobile-based application that is used to improve health and healthcare education. It develops awareness and skill set among the community health workers in India. The app was developed to complement the existing healthcare regime. The Pragati application is launched in Sonipur and Bhiswanath district in Assam. The underlying reason behind launching the app was Assam has the highest mortality rate in India. 237 women die out of 1,00,000 pregnancies at birth.

2.4) Mobile for Mothers Application - To improve the Quality of maternal healthcare, Welthungerhilfe's partnered with NEEDS has introduced a mobile Health Application in Jharkhand named Mobile for Mother's app in collaboration with Rural Health Mission. The software has been designed to support ASHA in Liptara block, one of the poorest areas of the state. Through the mobile app, it gets easy to register for advising on ANC and safe institutional delivery.

2.5) Upasana (Diagnostic toolkit for ASHA workers)-Upasana is a medical diagnostic toolkit non-invasive in nature designed to be used by the ASHA workers. Upasana transfers data to the doctors at the hospital for diagnosis and measures the vital parameters of the patients in rural areas.

2.6) CommCare App- CommCare operates through the use of locally available and inexpensive android smartphones. It supports ASHA by supporting by facilitating better data collection, support-decision, and access to educational training material. It improves the performance of ASHA by tracking data in real-time by managing cases. It takes into account the households they visit, quality of decision-making, and health outcomes of the household they serve. The mobile software includes checklists, registration forms, tools for monitoring danger signs, and educational media (images and videos) in multiple languages. It has shown ASHA's increase in retention of health-related knowledge and keep them updated about the scheduled visits. The app is used by over 50 organizations in 25 countries across the globe. It is created by Dimagi, a united state-based technology that is used by CHW and contributes to strengthening program management by providing customized data for decision-making.

2.7) IT services launched Mobile App- IT services in Hyderabad launched the mobile app to allow ASHA workers to access maternal healthcare. The mobile application is developed by Kelton Tech Solutions Ltd., launched in New Delhi to coincide with Andhra Pradesh. The 
DOI: $10.46523 /$ jarssc. 05.01 .09

Multidisciplinary, Open Access

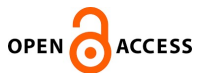

audio-visual feature allows to track the health parameters of women and children for the future. The workers are sensitized on danger signs in pregnancy that includes desired nutritional status, symptoms of high-risk pregnancy and timely immunization, etc.

2.8) MAMA (Mobile Alliance for Maternal Action): The Application is launched in partnership with USAID, Johnson \& Johnson, mhealth alliance. It involves female health care from pregnancy till childbirth. It includes sending personalized messages 2-3 times a week to prevent maternal health risks.

2.9) SMS One: The SMSOne is one of the business models that generate revenue SMS- based information services are targeted to local communities particularly large cities. The local community receives free of charge SMS while Local business is charged for advertising through SMS. In March 2009, SMSOne has more than 3,00,000 subscribers in Andhra Pradesh and Maharashtra.

2.10) TARSHI: The helpline received 57000 calls in its eleven years of operation. It offers a careful selection of inductive training, a careful selection of counselors, training, supervision, and reliable referral systems. It offers induction training, perspective building, close supervision, and reliable referral systems.

2.11) Mobile Academy: It is an interactive voice response-based application running on a Voice service delivery platform are integrated with mobile operators in Bihar, Airtel, Idea, Reliance, TATA, and Vodafone provides training course on maternal and child health for community health workers.

2.12) Manglam Sewa Samiti: The JSY helpline tracks pregnant women and motivates them for institutional delivery. The counselors use a checklist to assess complications and provide counseling. The application provides continuous advice to pregnant women and the delivery of service.

In this context, it is essential to understand the features of Digital Health applications. It consists of a variety of features that makes their usage easy and accessible to community health workers. firstly, the applications are kept simple to use and focused in nature so that community health workers perform actions without cognitive load and with ease. Secondly, they can work with zero connectivity for offline health workers also. Thirdly, they are either for personal usage or clinic-owned devices. Lastly, they are light on data usage. 
Journal of Advance Research in Science and Social Science (JARSSC)

Official Publication of Indian Mental Health \& Research Centre

DOI: $10.46523 /$ jarssc. 05.01 .09

Multidisciplinary, Open Access
ISSN: 2582-2004

Volume 05, Issue 01

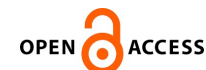

Crossref

In rural communities, ASHA workers are the primary contact for community women. The problem lies in undergoing training by health workers. It is considered substandard and erratic which leads to low retention among the ASHA's. The question arises as to How are online digital applications better than the traditional model of health communication? firstly lack of skillset and Education, Secondly Insufficient Training, Third Poor sharing of sensitive information and fourth non-engaging method of training. The objective behind providing the digital platform to share health information and awareness to the community women includes 1) They can impart experiences, knowledge, and learn from their peers instead of just trainingbased learning. 2) They can sense belongingness and be a part of the bigger community over the physical community 3) They are motivated to learn and explore new possibilities through digital interaction,4) They stay updated with campaigns and government schemes that serve the community.

2.8) ASHA Soft - This application in google pay is used to facilitate ASHA worker efficiency and timely payments which results in better coverage on healthcare-seeking beneficiaries from the public health system. It provides a platform for a periodic appraisal on performance and processing payments that will bring transparency in the workflow of the ASHA workers. The application is launched by the Haryana government. With this application, payment of monthly incentive amount and honorarium to ASHA workers is faster in digital mode.

Therefore, the mHealth Application launched to aid ASHA workers has transformed the Indian Healthcare system. However, there were challenges encountered in context to mHealth Applications. The Report 'Assisted Telemedicine for rural healthcare system' mentioned The Challenges as follows: Firstly, Poor Internet connectivity caused an interruption during consultation. The poor connection led to distraction and ASHA workers had to struggle with smooth connection. Secondly, Villagers were hesitant to use teleconsultation and use digital platforms. Thirdly, the trial was first conducted with patients in community health centers (CHC) and due to lack of time patients did not appear in trials. Lastly, government doctors were equipped with work so they didn't allow sufficient time for trial (Raj \& Srikanth,2020).

\section{Results}

The mobile applications are designed to reduce the burden of community health workers in advancing planning and communication skills. The tools were devised to ensure the quality of 
Journal of Advance Research in Science and Social Science (JARSSC)

Official Publication of Indian Mental Health \& Research Centre

DOI: $10.46523 /$ jarssc. 05.01 .09

Multidisciplinary, Open Access
ISSN: 2582-2004

Volume 05, Issue 01

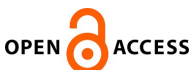

Crossref

counseling and institutional deliveries, regular antenatal checkups, vaccination on time, and post-natal care.

The Report Digital Health Trends stated that innovation in digital health tools includes mobile health apps, wearable sensors, and new approaches in context to the management of health conditions. The benefits of e-Health include firstly increase in affordable costs, secondly Enhancing and improving the efficiency of health care services, Third, By sharing knowledge to patients where decisions can be taken in a shared manner.

The findings based on the mobile applications that depicted the various trends in medical healthcare are:

1. Entries in Digital Mode - The traditional mode required registers to pen down the entries and now can be entered using the Mobile phone that is easy to operate and faster mode.

2. Pre-loaded Questionnaire- Software has a pre-installed questionnaire that allows ASHA's to discuss issues with beneficiaries. Responses can thus be entered.

3. Pop-up messages as alerts- The application activates pop-up messages as an alert if the pregnant woman faces a health issue The ASHA worker can thus facilitate timely help.

4. Audio-visuals linked with questions - The audio-visuals conduct efficient interpersonal communication sessions by linking with certain questions and creating health awareness. They deal with hemoglobin, antenatal care, and timely vaccinations for pregnant women.

5. Data Entry (Online and Offline) - ASHA's can solve the issue of net connectivity with online and offline data entry. It also avoids errors in the multi-handling of data.

6. Automatic Plan of action generation-ASHAs communicate better with the beneficiary by an automated generation of the next day's schedule. It tells the community women that $\mathrm{ANC} / \mathrm{PNC}$ appointment is due. The server generates it through automatic mode.

7. Repeated Pop-up alerts- Alerts are repeated pop-up messages that improve capacity of ASHA's about the health status of pregnant woman suffering from a medical condition or natural deficiency.

8. Authentic data generation and transmission - Updates from software helped in gathering accurate information from the respondents. The information is inserted and data is generated in good time. 
Journal of Advance Research in Science and Social Science (JARSSC)

Official Publication of Indian Mental Health \& Research Centre

DOI: $10.46523 /$ jarssc. 05.01 .09

Multidisciplinary, Open Access
ISSN: 2582-2004

Volume 05, Issue 01

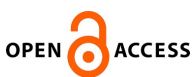

Crossref

\section{Conclusion}

The digital mobile applications have brought revamp in the traditional healthcare system. Technology has enabled India to leapfrog into a healthcare system that is advanced and fulfills the vision of the National Health Policy. ASHA's have been regarded as Digital disruptors due to real-time health monitoring through mobile health applications. The mobile health applications launched to facilitate ASHA workers in different states of the country have created a new field of eHealth. Mobile Phones are comparable to popular media and have been used for spreading healthcare awareness. Digital empowerment is necessary if in case the health worker faces problems in indecisive situations. The IT technology has enabled a model that integrates data capture from registration to tracking of service delivery and measuring the outcomes generated. Mobile technology involves testing and screening modalities replacing the traditional method of health communication. Technologies have voice-enabled records that emerged as a practice globally for improving the reliability of data. Thus, various trends in healthcare have added up to their skills and effectively transformed the Indian Healthcare system. Therefore, Digital enablement will not just add to their skills and upgrade their role as health communicators rather lead to more advancement in digital communication.

\section{References}

Arya, K. (2016). Information Communication Technology used by Health Care Worker (ASHA) of Uttarakhand. International Journal of Advanced Research, 1897-1902.

Assisting community health workers in India -Dimagi's Commcare. (2013). Innovation Catalyst, 13-18.

Chib, A., Jia, C. Y., Lynette, L. C., Hwee Cheryl, N. C., Kee, T. C., \& VLV, K. (2012). ASHA: The Hope of Information Communication Technologies in Indian Rural Healthcare. Academia, 1-17.

Digital healthcare: regulating the revolution. (2018). Biomed Journal.

Digital Transformation in Healthcare in 2021: 7 Key Trends. (2021, January 04). Retrieved from Digital Authority Partners: https://www.digitalauthority.me/resources/state-ofdigital-transformation-healthcare/

eHealth India. (2017, 05 29). Retrieved from National Health Portal: http://www.nhp.gov.in/ehealth-india_mty 
Journal of Advance Research in Science and Social Science (JARSSC)

Official Publication of Indian Mental Health \& Research Centre

DOI: $10.46523 /$ jarssc. 05.01 .09

Multidisciplinary, Open Access
ISSN: $2582-2004$

Volume 05, Issue 01

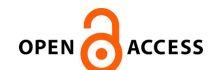

Crossref

BIBLIOGRAPHY $\backslash 11033$ 7) Eysenbach, G. (2001). What is e-health? Journal of Medical Internet Research, 3-91.

Gustafson, D. H., Robinson, T. N., Ansley, D., Adler, L., \& Brennan, P. F. (1999). Consumers and Evaluation of Interactive Health Communication Applications. American Journal of Preventive Medicine, 23-29.

Health Communication. (2021, January 13). Retrieved from Wikipedia: https://en.wikipedia.org/wiki/Health_communication

Improving healthcare through ICT for India's rural women: e-ASHA in Rajasthan. (2014). The International Journal for Rural Development.

IT Services Company Launches Mobile App to Allow ASHA Workers to Access Maternal HealthCare. (2015, 04 23). Retrieved from Tech Digital: https://www.franchiseindia.com/wellness/it-services-company-launches-mobile-appto-allow-asha-workers-to-access-maternal-healthcare.5520

Kohli Charu, K. J. (2015). Knowledge and Practice of Accredited Social Health activists for maternal healthcare delivery in Delhi. Journal of Family Medicine and Primary Care, $359-363$

BIBLIOGRAPHY $\backslash 116393$ Karahainko, K. (2020). The innovative future of Healthcare with the mHealth apps. Mentalstack.

Mapping mHealth Success: The ReMind Project. (2016). Catholic Relief Services, 1-12.

Mobile for Mothers: Improving capacities of health workers. (2018, May 23). Retrieved from Welthungerhilfe (For a world without hunger): https://welthungerhilfeindia.org/mobile-for-mothers-improving-capacities-of-healthworkers/

Murthy, D., \& Vijayaraman, D. (2012). Role of Mobile Health in Female Health Workers Health Routine. Mysore, Karnataka: FRHS, India.

mSakhi : Award-Winning Mobile Phone App. (n.d.). Retrieved from Intra Health: https://www.intrahealth.org/msakhi-award-winning-mobile-phone-app-frontlinehealth-care 
Journal of Advance Research in Science and Social Science (JARSSC)

Official Publication of Indian Mental Health \& Research Centre

DOI: $10.46523 /$ jarssc. 05.01 .09

Multidisciplinary, Open Access
ISSN: $2582-2004$

Volume 05, Issue 01

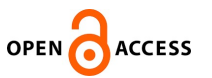

Rethinking Health in the E-health Era. (2003). Pubmed, 7-23. Retrieved from National Library of Medicine.

BIBLIOGRAPHY $\backslash 116393$ Rudolph, M., Barr, M. S., Kuhn, T., \& McDonald, C. J. (2008).

E-Health and Its Impact on Medical Practice. American College of Physicians, 1-25.

Shankar Prinja, G. A. (2018). Cost analysis of implementing mHealth intervention for maternal, newborn and child health care through community health workers: assessment of ReMIND program in Uttar Pradesh, India. BMC Pregnancy and Childbirth.

Shrivastava Archana, S. A. (2016). Measuring Communication Competence and effectiveness of ASHAs in their leadership role in rural settings of Uttar Pradesh. Leadership in Health Services, 69-81.

UPASANA: Diagnostic Toolkit for ASHA workers. (2015, November 21). Retrieved from IEEE Xplore: https://ieeexplore.ieee.org/document/74232919

Vij Launches an android application and Web Portal called ASHA-pay for digital Payment. (2020, October 23). Retrieved from United News of India: http://www.uniindia.com/vij-launches-an-android-application-web-portal-called-ashapay-for-digital-payment/north/news/2210733.html

S. Renata, (2013). Health Communication: From Theory to Practice (p. 656). New York: Wiley.

7 ways digitalization will shape the future of Healthcare. (2019, September 30). Retrieved from Healthcare Transformers: https:/healthcaretransformers.com/healthcarebusiness/digitalization-future-of-healthcare/

BIBLIOGRAPHY $\backslash 116393$ eHealth, when technology becomes the key to social wellbeing. Retrieved from Iberdrola: https:/www.iberdrola.com/innovation/ehealth 